\title{
The Effect of Rotenone on Ndfip1 in MES23.5 Cells
}

\author{
Xin Liu
}

\begin{abstract}
Parkinson's disease (PD) is a common degenerative disease of the nervous system. The pathogenesis of PD is not yet clear. However, it has been reported that many factors including age, environmental factors, and genetic factors are included. Rotenone is one of the naturally occurring insecticides found in many plants of the Derris, Lonchocarpus, Tephrosia and Mundulea species. It is also one of the classic neurotoxic drugs to produce PD models. Ndfip1 has been reported to be a neuroprotective protein in the brain. Therefore, in this study, we examined the expression of Ndfip1 in the mitochondrial complex I inhibitor rotenone-induced PD models in MES23.5 dopaminergic cells. Our results showed that rotenone has a concentration-dependent and time-dependent impairment effect on MES23.5 cells. When the concentration of rotenone was $25 \mathrm{nmol} / \mathrm{L}$, the viability of the cells was significantly decreased at $24 \mathrm{hrs}$. Further study showed that the expression of Ndfip 1 increased in the mRNA levels at $6 \mathrm{hrs}$ after $25 \mathrm{nmol} / \mathrm{L}$ rotenone treatment. The protein levels of Ndfip1 increased at $3 \mathrm{hrs}, 6 \mathrm{hrs}$ and decreased at $12 \mathrm{hrs}$ after $100 \mathrm{nmol} / \mathrm{L}$ rotenone treatment. This indicates that rotenone caused damage to MES23.5 dopaminergic cells, which is accompanied by a decrease of Ndfip1.
\end{abstract}

Keyword: Parkinson’ Disease; MES23.5 Cells; Rotenone; Ndfip1

\begin{abstract}
Introduction
Parkinson's disease (PD) is a common neurodegenerative disease and was first reported by British physician James Parkinson in 1817. The characteristic clinical manifestations of PD are resting tremor, muscle rigidity, bradykinesia, and postural instability[1]. The etiology and pathogenesis of PD have not yet been elucidated. At present, it is believed that it may be related to many factors such as heredity, environment, oxidative stress, mitochondrial dysfunction, excitatory toxins, and apoptosis, and is the result of a combination of multiple mechanisms[2-7]. Until now, levodopa replacement therapy is the main treatment of PD. However, this often induces dyskinesia and other adverse reactions after being used for 4 to 5 years, and it is difficult to overcome. Therefore, to study the pathogenesis of PD might provide new perspective for the treatment of PD.
\end{abstract}

Rotenone is a natural compound derived from the root of the Lonchocarpus species and is used as an insecticide and herbicide[8]. It is a highly lipophilic compound that easily crosses the blood-brain barrier[9]. Rotenone irreversibly binds to complex I of the mitochondrial electron transport chain to induce the selective loss of nigral dopaminergic neurons and further induce Parkinson-like symptoms[10, 11]. It has been suggested that mitochondrial dysfunction might be involved in the pathogenesis of PD. The decreased activity of complex I in the brain tissue, muscle, and platelet of patients with PD further supports this hypothesis[12]. Therefore, rotenone treatment can mimic the clinical and pathological features of $\mathrm{PD}$, and it may be a better tool for the study of the pathogenesis of PD. In this study, the PD cell model established by rotenone was used to explore the changes in the expression of Ndfip1.

Ndfip1 was first reported in 2000 by the Far-Western blot[13] and has a molecular weight of approximately $26 \mathrm{kDa}$ with 221 amino acid residues. Ndfip1 protein has received extensive attention for its unique ability to bind to the Nedd 4 family of proteins[14]. It has been reported that the expression of Ndfip1 protein increased in the neurons of traumatic brain injury and cerebral ischemia[14, 15], indicating its neuroprotective effect. In this study, we explored the mRNA and protein expression of Ndfip1 in rotenone-treated MES23.5 cells. This provides a new target for drug intervention for the prevention and treatment of PD.

\section{Materials and methods \\ Chemical reagents}

The MES23.5 dopaminergic cell line was gifted by Professor Weidong Le of Dalian Medical University. The primary antibody of Ndfip1 was purchased from Sigma (St. Louis, USA). The monoclonal $\beta$-actin antibody was from Bioss (Beijing, China). The goat anti-rabbit IgG labeled with HRP was from Santa Cruze (Dallas, USA). Fetal bovine serum (FBS) and DMEM/F-12 were from Hyclone (Logan, Utah, USA). Penicillin-streptomycin solution was purchased from Beyotime (Shanghai, China). MTT, dimethyl sulfoxide (DMSO) and the reagents used for Sato's solution were purchased from Sigma (St. Louis, USA). TRIZOL was brought from Invitrogen (USA). Other biological reagents and materials are from local commercial sources.

This article is published under the terms of the Creative Commons Attribution License 4.0

Author(s) retain the copyright of this article. Publication rights with Alkhaer Publications.

Published at: http://www.ijsciences.com/pub/issue/2018-05/

DOI: 10.18483/ijSci.1659; Online ISSN: 2305-3925; Print ISSN: 2410-4477 


\section{Cell culture and treatment}

The cell culture medium containing 10\% FBS and $1 \%$ penicillin-streptomycin solution was first heated to $37^{\circ} \mathrm{C}$. Rinse cell culture flasks or plates with 1 $\mathrm{mg} / \mathrm{mL}$ polylysine and remove them, then rinse the flasks or plates again with $0.01 \mathrm{M}$ PBS. The MES23.5 cells were then seeded in a cell culture flask or plate at a density of $1 \times 10^{5} / \mathrm{cm}^{2}$ and cultured in an incubator containing $37^{\circ} \mathrm{C}$ and $5 \% \mathrm{CO}_{2}$.

\section{MTT assay for cell viability}

MES23.5 cells were plated in 96-well plates at a density of $1 \times 10^{5} / \mathrm{cm}^{2}$. When the cell density reached $50 \sim 70 \%$, MES23.5 cells were treated with $25 \mathrm{nM}, 50$ $\mathrm{nM}, 100 \mathrm{nM}, 200 \mathrm{nM}, 400 \mathrm{nM}$, or $800 \mathrm{nM}$ rotenone for 24 hours. To further study the effect of rotenone exposure on the cell viability of MES23.5 cells at different time points, cells were exposed to $25 \mathrm{nM}$ rotenone for $6 \mathrm{hrs}, 12 \mathrm{hrs}, 24 \mathrm{hrs}$, and $36 \mathrm{hrs}$ respectively.

The culture medium was removed and $20 \mu$ of sterile MTT solution $(5 \mathrm{mg} / \mathrm{mL})$ was added to each well and incubated in the incubator for $4 \mathrm{hrs}$. Then the supernatant was discarded, and $100 \mu \mathrm{l}$ of DMSO was added to each well and shaken on a shaker for 10 minutes to fully dissolve the crystals. Using an automatic microplate reader to measure absorbance $(A)$ at $490 \mathrm{~nm}$, calculate cell viability. Cell viability = $A_{\text {experimental group }} / A_{\text {control group }} \times 100 \%$.

\section{Real-time fluorescence quantitative polymerase chain reaction $(\mathbf{P C R})$}

Total RNA was isolated from the MES23.5 cells using the Trizol reagent. A total of $1 \mu \mathrm{g}$ of total RNA was transcribed into cDNA. All PCRs were performed using a fluorescence quantitative PCR instrument (Eppendorf) in a total volume of $20 \mu \mathrm{L}$. GAPDH serves as an internal control. Use the following primer sequences: Ndfip1 forward: 5'-CATCGAATCATTAGTGGTTA-3'; Ndfip1 reverse: 5'-GATGGAGGATGAATAAAGC-3'. GAPDH forward:

5'-CCCCCAATGTATCCGTTGTG-3'; GAPDH reverse: 5'-GTAGCCCAGGATGCCCTTTAGT-3'. From each amplification plot, a threshold cycle $(\mathrm{Ct})$ value and the numbers of transcripts were calculated.

\section{Western blotting}

MES23.5 cells were washed with ice-cold PBS and lysed in lysis buffer containing $50 \mathrm{mM}$ of Tris $\mathrm{HCl}$, $150 \mathrm{mM}$ of $\mathrm{NaCl}, 1 \%$ Nonidet $\mathrm{P}-40,0.5 \%$ sodium deoxycholate, $1 \mathrm{mM}$ of EDTA, $1 \mathrm{mM}$ of phenylmethylsulfonyl fluoride (PMSF), and protease inhibitors $(1 \mathrm{~g} / \mathrm{mL}$ of pepstatin, $1 \mathrm{~g} / \mathrm{mL}$ of aprotinin, and $1 \mathrm{~g} / \mathrm{mL}$ of leupeptin). The lysates were centrifuged at $12,000 \times \mathrm{g}$ for 10 minutes, and the protein concentration of the supernatants was determined by a BCA assay kit (Beyotime Biotechnology, China). A total of $20 \mu \mathrm{g}$ of protein was run on a $12 \%$ SDS polyacrylamide gel and transferred to a PVDF membrane $(300 \mathrm{~mA}, 100$ minutes). After blocking with 5\% skim milk overnight at $4^{\circ} \mathrm{C}$, the membranes were incubated with Ndfip1 antibody for 4 hours at room temperature. The blot was probed simultaneously with an anti- $\beta$-actin monoclonal antibody as a loading control The cross-reactivity was visualized using ECL western blotting detection reagents and analyzed by scanning densitometry by UVP imaging system.

\section{Statistical analysis}

The experimental results were processed using Prism 5.0 statistical software (GraphPad Software, Inc., San Diego, CA). Data were analyzed statistically using one-way ANOVA. $P<0.05$ was considered to be statistically significant.

\section{Result}

1. The effect of rotenone on cell viability by MTT assay in MES23.5 cells

MES23.5 cells were seeded on 96-well plates and were treated with $0 \mathrm{nM}, 25 \mathrm{nM}, 50 \mathrm{nM}, 100 \mathrm{nM}, 200$ $\mathrm{nM}, 400 \mathrm{nM}$, or $800 \mathrm{nM}$ rotenone for 24 hours. The cell viability was assayed using MTT. Results showed that the cell viability decreased significantly with the increase of rotenone concentration. The cell viability decreased from $25 \mathrm{nM}$ rotenone treatment and further reduced when the rotenone concentration increased, compared with the control group (Fig.1A).

We further studied the effect of $25 \mathrm{nM}$ rotenone treatment on the cell viability of MES23.5 cells at different time points. The results showed that the cell viability decreased significantly at $24 \mathrm{hrs}$ and further decreased at $36 \mathrm{hrs}$ after exposure to $25 \mathrm{nmol} / \mathrm{L}$ rotenone, compared with the control group (Fig.1B). 
A

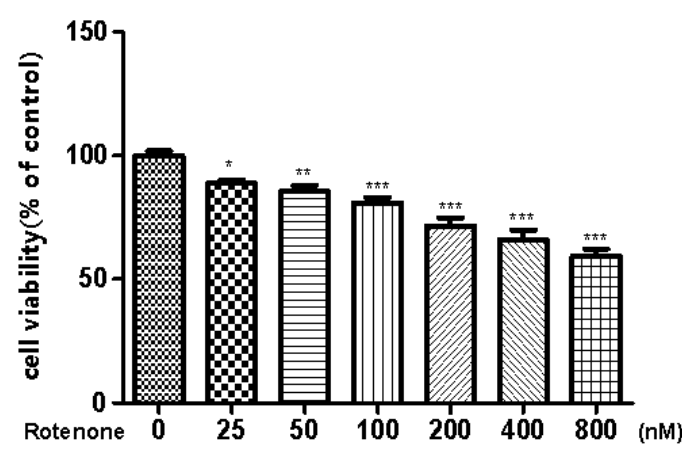

B

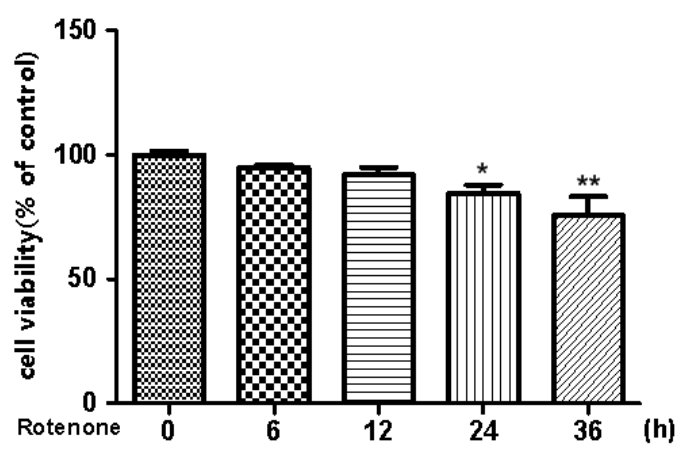

Fig.1 Rotenone induced the damage of MES23.5 cells in a concentration- and time- dependent manner.

A. The cell viability of rotenone-treated MES23.5 cells decreased from $25 \mathrm{nM}$ to $800 \mathrm{nM}\left({ }^{*} P<0.05,{ }^{* *} P<0.01\right.$, ${ }^{* * * *} P<0.001$, compared with the control).

B. Following treatment with $25 \mathrm{nM}$ rotenone, cell viability of MES23.5 cells decreased at 24 hrs and $36 \mathrm{hs}\left({ }^{*} P<0.05\right.$, ${ }^{* *} P<0.01$, compared with the control).

\section{The expressions of Ndfip1 decrease in MES23.5} cells after exposure to rotenone

We studied the mRNA expression of Ndfip1 in MES23.5 cells exposed to rotenone at different time points. Following treatment with $100 \mathrm{nmol} / \mathrm{L}$ rotenone, the mRNA levels of Ndfip1 were quantified in MES23.5 cells by real-time PCR at 3 hrs, 6 hrs, 9 hrs, 12 hrs and 24 hrs. Results showed that rotenone could increase the mRNA expression of Ndfip1 at 6 hs, compared with the control (Fig. 2). In parallel, we also detected the protein levels of Ndfip1 in rotenone-treated MES23.5 cells by western blots. Data showed that the protein expression of Ndfip1 increased significantly at $3 \mathrm{hrs}$ and $6 \mathrm{hrs}$, and decreased at $12 \mathrm{hrs}$ after rotenone treatment in MES23.5 cells, compared with the control (Fig. 3).

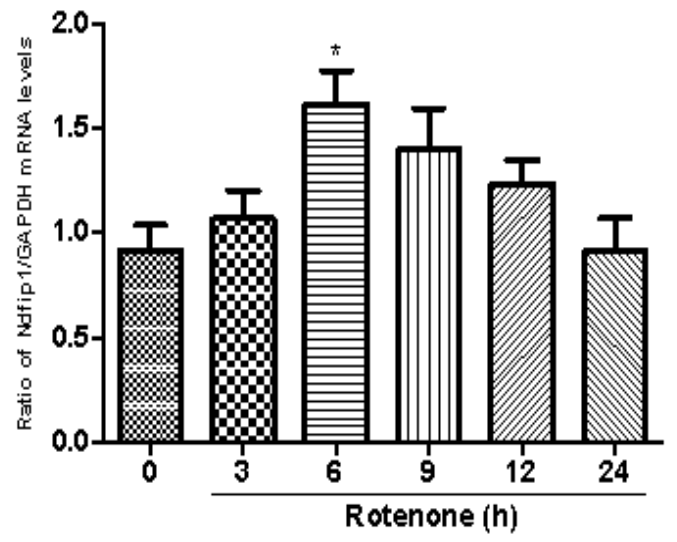

Fig. 2 The mRNA and protein expressions of Ndfip1 after exposure to rotenone in MES23.5 cells.

The mRNA expression of ndfip1 in rotenone-treated MES23.5 cells increased at $3 \mathrm{hrs,} \mathrm{compared} \mathrm{with} \mathrm{the}$ control ( $P<0.05$, compared with the control).
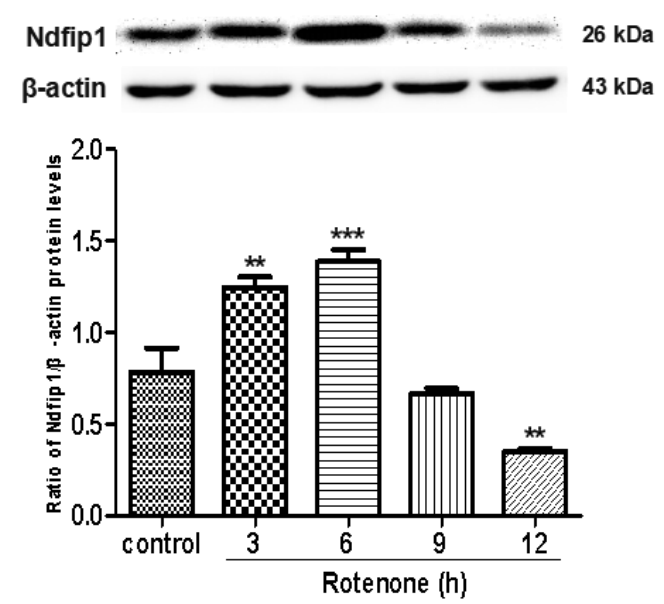

Fig. 3 The protein expressions of Ndfip1 after exposure to rotenone in MES23.5 cells.

The protein expression of Ndfip1 in rotenone-treated MES23.5 cells increased at $3 \mathrm{hrs}$ and $6 \mathrm{hrs}$ and decreased at $12 \mathrm{hrs}\left({ }^{* * *} P<0.01,{ }^{* * *} P<0.001\right.$, compared with the control).

\section{Discussion}

Rotenone is a highly selective inhibitor of mitochondrial respiratory chain complex I[16]. It was reported that the toxicity of rotenone is dependent on the interaction with complex I, and rotenone-induced neurotoxicity can be attenuated by antioxidants, indicating a causal role of oxidative damage[17]. Further experimental results showed that rotenone not only produced a major inhibitory effect on the electron transport activity of complex I, but also produced a secondary mechanism by increasing superoxide free radical production. This was responsible for the further development of structural and functional damage to mitochondria[18]. Results also showed that rotenone toxicity in vivo not only led to a significant decrease in the activity of 
complex I, but also induced the decrease in the activity of complex II mediated by ROS[19].

Animal models induced by chronic systemic administration of rotenone can present clinical and pathological features similar to those of human PD, including selective chronic and progressive degeneration of the nigrostriatal system and the formation of inclusion bodies similar to Lewy bodies[20]. It also induces oxidative stress and inhibition of mitochondrial complex I found in human PD patients[9, 17, 21, 22]. After rotenone enters the cell, aggregation occurs in the subcellular organelles including mitochondria. Among them, rotenone has a strong affinity for mitochondrial complex I, which binds to the PSST subunit of complex I polypeptidase and can selectively block the role of iron-sulfur clusters N2 and ubiquinone $\mathrm{Q}[23]$. The decreased activity of mitochondrial complex I stimulates the production of reactive oxygen species and aggravates oxidative stress. This can lead to abnormal mitochondrial function, which ultimately leads to decrease in cell viability[24]. In this study, we treated MES23.5 cells with rotenone and measured the cell viability by MTT assay. Our results showed that exposure of rotenone induced a decrease in the cell viability of MES23.5 cells and showed a time-dependent and concentration-dependent manner. This result is also consistent with the experimental results of Ferrante et $\operatorname{al}[24]$.

Increasing evidence showed that Ndfip1 is a neuroprotective protein and Ndfip1/Nedd4 ubiquitination pathway is activated during traumatic brain injury and cerebral ischemia[25-27]. Ndfip1 is also recognized as an adaptor and activator of the Nedd4 family of proteins[28]. It was also reported that Ndfip1 might serve as an early sensory protein for the ubiquitination of certain proteins to eliminate harmful misfolded proteins in injured neurons, promote the survival of neurons and play an anti-apoptotic effect[29]. In our experiments, the mRNA expression of Ndfip1 was up-regulated at 6 hrs after treatment with rotenone in a rotenone-induced PD cell model. Data also showed that the protein expression of Ndfip1 increased significantly at $3 \mathrm{hrs}$ and $6 \mathrm{hrs}$ and decreased at 12 hrs after rotenone treatment in MES23.5 cells, compared with the control. The results indicated that Ndfip1 protein might play a neuroprotective role in inhibiting apoptosis and promoting the survival of dopaminergic cells to counteract the toxic effects of rotenone on MES 23.5 dopaminergic cells. And the decreased expression of Ndfip1 might be involved in the pathogenesis of PD. However, the underlying mechanisms of the neuroprotective effect of Ndfip1 on rotenone-induced neurotoxicity should be further studied in the future.
In summary, our results demonstrated the changes in the expression of Ndfip1 in MES23.5 dopaminergic cells exposed to rotenone both in the mRNA and the protein levels. To explore the relationship between rotenone and Ndfip1 will provide evidence to reveal the pathogenesis of PD and provide a new therapeutic target for the development of new therapeutic drugs.

\section{References}

1. Jankovic, J., Parkinson's disease: clinical features and diagnosis. J Neurol Neurosurg Psychiatry, 2008. 79(4): p. 368-76.

2. Halliwell, B., Reactive oxygen species and the central nervous system. J Neurochem, 1992. 59(5): p. 1609-23.

3. Lee, V.M. and J.Q. Trojanowski, Mechanisms of Parkinson's disease linked to pathological alpha-synuclein: new targets for drug discovery. Neuron, 2006. 52(1): p. 33-8.

4. Lin, M.T. and M.F. Beal, Mitochondrial dysfunction and oxidative stress in neurodegenerative diseases. Nature, 2006. 443(7113): p. 787-95.

5. Takahashi, R., J. Kawamata, and H. Takeuchi, [Pathogenesis of sporadic Parkinson's disease: contribution of genetic and environmental risk factors]. Rinsho Shinkeigaku, 2009. 49(11): p. 885-7.

6. Zecca, L., et al., Iron, brain ageing and neurodegenerative disorders. Nat Rev Neurosci, 2004. 5(11): p. 863-73.

7. Zhang, Y., V.L. Dawson, and T.M. Dawson, Oxidative stress and genetics in the pathogenesis of Parkinson's disease. Neurobiol Dis, 2000. 7(4): p. 240-50.

8. Uversky, V.N., Neurotoxicant-induced animal models of Parkinson's disease: understanding the role of rotenone, maneb and paraquat in neurodegeneration. Cell Tissue Res, 2004. 318(1): p. 225-41.

9. Betarbet, R., et al., Chronic systemic pesticide exposure reproduces features of Parkinson's disease. Nat Neurosci, 2000. 3(12): p. 1301-6.

10. Manning-Bog, A.B., et al., The herbicide paraquat causes up-regulation and aggregation of alpha-synuclein in mice: paraquat and alpha-synuclein. J Biol Chem, 2002. 277(3): p. 1641-4.

11. Seniuk, N.A., W.G. Tatton, and C.E. Greenwood, Dose-dependent destruction of the coeruleus-cortical and nigral-striatal projections by MPTP. Brain Res, 1990. 527(1): p. 7-20.

12. Schapira, A.H., et al., Mitochondrial complex I deficiency in Parkinson's disease. J Neurochem, 1990. 54(3): p. 823-7.

13. Jolliffe, C.N., et al., Identification of multiple proteins expressed in murine embryos as binding partners for the WW domains of the ubiquitin-protein ligase Nedd4. Biochem $\mathrm{J}$, 2000. 351 Pt 3: p. 557-65.

14. Harvey, K.F., et al., N4WBP5, a potential target for ubiquitination by the Nedd4 family of proteins, is a novel Golgi-associated protein. J Biol Chem, 2002. 277(11): p. 9307-17.

15. Sang, Q., et al., Nedd4-WW domain-binding protein 5 (Ndfip1) is associated with neuronal survival after acute cortical brain injury. J Neurosci, 2006. 26(27): p. 7234-44.

16. Xiong, N., et al., Mitochondrial complex I inhibitor rotenone-induced toxicity and its potential mechanisms in Parkinson's disease models. Crit Rev Toxicol, 2012. 42(7): p. 613-32. 
17. Sherer, T.B., et al., Mechanism of toxicity in rotenone models of Parkinson's disease. J Neurosci, 2003. 23(34): p. 10756-64.

18. Domingues, H.S., et al., Oligodendrocyte, Astrocyte, and Microglia Crosstalk in Myelin Development, Damage, and Repair. Front Cell Dev Biol, 2016. 4: p. 71.

19. Panov, A., et al., Rotenone model of Parkinson disease: multiple brain mitochondria dysfunctions after short term systemic rotenone intoxication. J Biol Chem, 2005. 280(51): p. 42026-35.

20. Garcia-Garcia, F., et al., Sleep disturbances in the rotenone animal model of Parkinson disease. Brain Res, 2005. 1042(2): p. $160-8$.

21. Sherer, T.B., et al., An in vitro model of Parkinson's disease: linking mitochondrial impairment to altered alpha-synuclein metabolism and oxidative damage. J Neurosci, 2002. 22(16): p. 7006-15.

22. Liu, L., et al., Oxidative stress contributes to arsenic-induced telomere attrition, chromosome instability, and apoptosis. J Biol Chem, 2003. 278(34): p. 31998-2004.

23. Schuler, F. and J.E. Casida, Functional coupling of PSST and ND1 subunits in NADH:ubiquinone oxidoreductase established by photoaffinity labeling. Biochim Biophys Acta, 2001. 1506(1): p. 79-87.

24. Ferrante, R.J., et al., Systemic administration of rotenone produces selective damage in the striatum and globus pallidus, but not in the substantia nigra. Brain Res, 1997. 753(1): p. 157-62.

25. Howitt, J., et al., Ndfip1 regulates nuclear Pten import in vivo to promote neuronal survival following cerebral ischemia. $\mathrm{J}$ Cell Biol, 2012. 196(1): p. 29-36.

26. Lackovic, J., et al., Differential regulation of Nedd4 ubiquitin ligases and their adaptor protein Ndfip1 in a rat model of ischemic stroke. Exp Neurol, 2012. 235(1): p. 326-35.

27. Low, L.H., et al., Nedd4 family interacting protein 1 (Ndfip1) is required for ubiquitination and nuclear trafficking of BRCA1-associated ATM activator 1 (BRAT1) during the DNA damage response. J Biol Chem, 2015. 290(11): p. 7141-50.

28. Liu, K., et al., Protective effects of Ndfip1 on MPP(+)-induced apoptosis in MES23.5 cells and its underlying mechanisms. Exp Neurol, 2015. 273: p. 215-24.

29. Myat, A., et al., Drosophila Nedd4, a ubiquitin ligase, is recruited by Commissureless to control cell surface levels of the roundabout receptor. Neuron, 2002. 35(3): p. 447-59. 